(all races) with stage IV melanomas captured in the NCDB for the periods from 2004-2011 and 2012-2016.

Conclusions Asian patients with melanoma are receiving diagnoses at older ages. Despite decreases in OS for all Asian patients with melanoma, advanced stage IV of the diseases have improved outcomes for the group treated in the era of ICI and TT. Further investigation is warranted to understand the treatment, patient, and tumor characteristics that predict response in this demographic of patients.

http://dx.doi.org/10.1136/jitc-2020-SITC2020.0729

\section{HISTOTRIPSY FOCUSED ULTRASOUND ABLATION INDUCES IMMUNOLOGICAL CELL DEATH IN TREATED AND DISTANT UNTREATED TUMORS}

Anutosh Ganguly*, Ashley Pepple, Reliza McGinnis, Ryan Hubbard, Amy Felsted, Tejaswi Worlikar, Hannah Garavaglia, Joe Dib, Joe Guy, Michal Olszewski, Zhen Xu, Clifford Cho. University of Michigan Medical School, Ann Arbor, MI, USA

Background Histotripsy, a novel image-guided, robotically assisted sonic therapy platform, is a non-invasive and nonthermal tumor ablation modality. We have previously shown that histotripsy potentiates profound innate and adaptive antitumor responses in addition to direct tumor destruction. ${ }^{1}$ In this study, we sought to characterize the biomarkers of tumor cell death pathways immediately after histotripsy and after the induction of adaptive anti-tumor immune responses in preclinical settings.

Methods Immunocompetent C57BL/6 mice were inoculated with bilateral subcutaneous flank injections of Hepa1-6 hepatocellular carcinoma to generate $8-10 \mathrm{~mm}$ tumors within 811 days. Unilateral subtotal histotripsy was then performed. Mice were euthanized at $6 \mathrm{~h}$, and 1,3 and 10-12 days posttreatment (dpt). Tumors were measured, harvested, fixed, sectioned and studied using multicolor immunohistochemistry.

Results Histotripsy decreased treated tumor growth by $50 \%$ and abscopal tumor growth by $30-40 \%$ compared to untreated tumors at $12 \mathrm{dpt}$, evidencing a systemic anti-tumor immune response that inhibited growth of distant untreated tumor. Treated tumors showed immediate tissue liquefaction in the ablation zone with marked extranuclear translocation of the damage associated molecular pattern HMGB1. At 1dpt, $100 \%$ of tumor cells within the ablation zone showed HMGB1 translocation, and $70 \%$ of tumor cells at the periphery of the ablation zone showed HMGB1 translocation. Caspase 3 cleavage was not observed in the direct ablation zone, but at the junction of the ablated and non-ablated tissue $\sim 40 \%$ cells that released HMGB1 showed cleaved Caspase 3. Caspase 9 cleavage was observed in $\sim 50 \%$ cells that had cleaved Caspase 3, suggesting early programed cell death with mitochondrial damage and cytochrome $\mathrm{C}$ release $1 \mathrm{dpt}$; the presence of inflammasome integration/activation suggested pyroptosis induction. Areas of tumor well outside the zone of ablation and within untreated tumors contralateral to ablated tumors did not show early DAMP release or apoptotic cell death compared to the control tumors. However, a robust immune cell infiltration was observed in these locations at 10-12dpt, involving CD8 T-cell infiltration and areas of tumoral HMGB1 release in the vicinity of the infiltrating CD8 $\mathrm{T}$ cells - indicating the induction of immune rejection of treated and untreated tumors by histotripsy.

Conclusions Our results indicate that histotripsy ablation promotes tumor cell destruction through both immediate mechanical disruption, as well as possible adjacent apoptotic and pyroptotic death. Systemic CD8 T-cell mobilization and immunological cell death in the treated and the contralateral tumors is a novel long term therapeutic benefit.

\section{REFERENCE}

1. Qu S, Worlikar T, Felsted AE, Ganguly A, Beems MV, Hubbard R, Pepple AL, Kevelin AA, Garavaglia H, Dib J, Toma M, Huang H, Tsung A, Xu Z, Cho CS. Non-thermal histotripsy tumor ablation promotes abscopal immune responses that enhance cancer immunotherapy. J Immunother Cancer 2020;8:e000200.

http://dx.doi.org/10.1136/itc-2020-SITC2020.0730

\section{Regulatory, financial, and access considerations}

\section{COSTS OF CARE FOR FIRST-LINE (1L) TREATMENT OF ADVANCED NON-SMALL CELL LUNG CANCER (ANSCLC): A REAL-WORLD CLAIMS ANALYSIS}

${ }^{1}$ Jonathan Kish*, ${ }^{1}$ Dhruv Chopra, ${ }^{1}$ Djibril Liassou, ${ }^{2}$ Solomon Lubinga, ${ }^{2}$ John Hartman, ${ }^{1}$ Bruce Feinberg. 'Cardinal Health, Dublin, OH, USA; ${ }^{2}$ Bristol Myers Squibb, Lawrenceville, NJ, USA

Background Recent advances in therapy have created numerous options for the $1 \mathrm{~L}$ treatment of aNSCLC. This study describes the total direct healthcare costs for patients treated with immunotherapy monotherapy (IO), chemotherapy (CT), or immunotherapy plus chemotherapy $(\mathrm{IO}+\mathrm{CT})$ in the $1 \mathrm{~L}$ setting.

Methods The Ability Patient Complete claims database was used to identify US patients aged $\geq 18$ years diagnosed with aNSCLC (ICD-9: 162.*; ICD-10: C34.*) initiating 1L treatment with IO, CT, or IO+CT between January 2015 and May 2019. Patients were required to have at least 6 months of continuous enrollment prior to initiation of $1 \mathrm{~L}$ treatment, $\geq 1$ inpatient or 2 outpatient claims for lung cancer, and a claim within 45 days for a secondary metastatic site. Patients with another malignant primary cancer, who participated in a clinical trial, or who received treatments consistent with small cell lung cancer or a systemic therapy not used for lung cancer were excluded. Costs were calculated on a per-patient per month (PPPM) basis from initiation of $1 \mathrm{~L}$ treatment until discontinuation or end of study period and expressed in 2019 US dollars. A standardized cost approach was applied, with average wholesale prices for antineoplastic and other drug costs and CMS fee schedules for outpatient visits, inpatient stays, ED visits, and other medical costs (e.g. all other outpatient medical services including infusions of growth factors, radiographic studies, blood draws, etc.). All antineoplastic costs were considered individually.

Results 8,154 patients were included in the cohort: 1,319 received $\mathrm{IO}, 5,315 \mathrm{CT}$, and 1,520 $\mathrm{IO}+\mathrm{CT}$. By cohort, mean age was $65(\mathrm{IO}), 63(\mathrm{CT})$, and $62(\mathrm{IO}+\mathrm{CT})$ years while mean Charlson Comorbidity Index was 2.12, 2.11, and 1.83, respectively. Key results by healthcare resource utilization category are provided in the table below (table 1).

Conclusions The total PPPM healthcare costs of patients receiving chemotherapy (CT or $\mathrm{IO}+\mathrm{CT}$ ) are higher than those only receiving IO monotherapy. These differences are driven by higher outpatient visit, other medical, and pharmacy costs. IO-containing regimens have higher antineoplastic costs than 